\title{
FERTILIZACIÓN Y DENSIDAD DE POBLACIÓN EN GENOTIPOS DE MAÍZ CULTIVADOS BAJO CONDICIONES DE TEMPORAL ${ }^{1}$
}

\author{
Octavio Cano ${ }^{2}$, Oscar Hugo Tosquy ${ }^{3}$, Mauro Sierra ${ }^{2}$, Flavio Antonio Rodríguez ${ }^{2}$
}

\begin{abstract}
RESUMEN
Fertilización y densidad de población en genotipos de maíz cultivados bajo condiciones de temporal. Durante el ciclo Primavera - Verano1998/98 se condujo una investigación en el Campo Experimental Cotaxtla, Veracruz, México, con los objetivos de conocer la respuesta de cinco genotipos de maíz a la fertilización y densidad de población. El experimento se estableció bajo un diseño de bloques al azar con dos repeticiones y un arreglo de tratamientos en parcelas subdivididas, donde la parcela chica fueron los genotipos (G): VS536, H-512, H-513, HTV-1 y HTV-2, las parcelas medias los tratamientos de fertilización (F) de $\mathrm{N}_{2}, \mathrm{P}_{2} \mathrm{O}_{5}, \mathrm{~K}_{2} \mathrm{O}:$ : 161-46-0, 184-69-0, 184-69-30, 207-92-0 y 207-92-30 y las parcelas grandes las densidades (D): 50; 62,5; 75; 93,75; 100 y 125 miles de $\mathrm{pl} / \mathrm{ha}$. Las variables estudiadas fueron el índice de cosecha, el porcentaje de plantas jorras (sin mazorca) y rendimiento de grano. Los análisis de varianza mostraron significancia para algunas variables en D, F, DxF y DxG y para todas en G. De acuerdo a lo anterior, el mejor genotipo fue el híbrido promisorio HTV-1 con rendimiento de 6,74 t/ha. La respuesta de fertilización sólo se dio en el índice de cosecha, donde con el tratamiento 184-69-0 se obtuvo el mayor valor $(0,446)$. Con $62500 \mathrm{pl} / \mathrm{ha}$ todos los genotipos presentaron su mayor rendimiento y bajos porcentajes de plantas "jorras".
\end{abstract}

\begin{abstract}
Fertilization and plant density in genotypes of corn under rainy season conditions. During the rainy season in 1998/98 a reseach was conducted in Campo Experimental Cotaxtla, Veracruz, México, with the objectives of knowing the response of five corn genotypes to doses fertilizer and plant density. A completed randomized block experimental design with two replications was used arranged in split plots where the genotypes (G) VS-536, H-512, H-513, HTV1 and HTV2 were considered as small plots ; The medium plots were doses of fertilization $(\mathrm{F})$ of $\mathrm{N}_{2}, \mathrm{P}_{2} \mathrm{O}_{5}, \mathrm{~K}_{2} \mathrm{O}$ : 161-46-0, 184-69-0, 184-69-30, 207-92-0 and 207-92-30 ; and the big plots were plant densities(D): 50, 62.5, 75, 93.75, 100 and 125 thousand plants/hectare. The characteristics studied were: harvest index, ears per plant, and grain yield. The analysis of variance showed significant differences for some characteristics in D, F, DxF y DxG and for all in G. According to this, the best genotype was the experimental hybrid HTV1 with yield $6.74 \mathrm{t} / \mathrm{ha}$. The response to fertilization was only of to harvest index, where the best dose was 184-69-0. In relation with plant density, with 62500 plants/hectare, all genotypes showed their best yield and number of ears per plant.
\end{abstract}

\section{INTRODUCCION}

En México, el maíz es uno de los cultivos de mayor importancia en la agricultura, la economía nacional y desde el punto de vista social, ya que en este cereal se basa un alto porcentaje la alimentación de sus habitan- tes. Por lo que poco más de 7,5 millones de hectáreas (40\% de la superficie agrícola) son sembradas año con año (Tosquy 1997).

De esta superficie poco más de tres millones de hectáreas se cultivan en el trópico húmedo, donde des-

\footnotetext{
${ }_{1}^{1}$ Recibido para publicación el 13 de abril del 1999. Trabajo presentado en la XLV Reunión Anual del PCCMCA. Guatemala, Guatemala, C.A., abril de 1999.

2 Programa de Maíz del Campo Experimental Cotaxtla. INIFAP. A.P. 429 Veracruz, Ver. México.91700. E-mail: cecot54@ver1.telmex.net.mx

3 Programa de Arroz del Campo Experimental Cotaxtla. CIRGOC. INIFAP, México.
} 
taca el estado de Veracruz con una superficie de siembra que supera las 530 mil hectáreas y un rendimiento medio que no alcanza las $2 \mathrm{t} / \mathrm{ha}$, debido entre otros factores, a que el $80 \%$ de la superficie se da bajo condiciones de temporal.

Las razones anteriores, la imperiosa necesidad de satisfacer la demanda de productos alimenticios básicos de una creciente población tanto estatal como nacional y el incremento constante de los insumos agrícolas que ocasionan que el costo de producción del cultivo de maíz sea muy alto, justifican los programas de investigación y mejoramiento en maíz tendientes a incrementar su producción por unidad de superficie y hacer más rentable el cultivo para que los productores maiceros de temporal en el estado continúen sembrando (Castañón et al. 1996).

Una de las formas de lograr lo arriba señalado es de combinar eficientemente factores tales como: dosis de fertilización, densidades de población, uso de semillas mejoradas, entre otros. El nitrógeno y el fósforo son los elementos que con mayor frecuencia limitan la obtención de altos rendimientos y en maíz no es la excepción, ya que varios estudios indican que una adecuada aplicación de estos macronutrimentos al suelo redituará en un incremento en el rendimiento de grano y principalmente en el aspecto proteínico.

De potasio en maíz INPOFOS (s.f.) menciona que un alto nivel de este elemento puede reducir el número de plantas estériles por hectárea, en especial donde se han usado altas densidades de plantas en condiciones de deficiencia de este nutrimento, además de mejorar el número de mazorcas llenas, de grano por mazorca y el peso de cada grano.

De acuerdo con Marín et al. (1989) es posible que con sólo incrementar la densidad de plantas por hectárea se logre producir más grano por unidad de superficie. Sin embargo, no todos los genotipos presentan la misma respuesta a altas densidades de plantas o dosis de fertilización, por ello se hace necesario estudiar a estos en cada cultivar que es promisorio o que está en camino de ser liberado. Por lo anterior, el objetivo del presente trabajo fue estudiar el efecto de la densidad de población y la fertilización en cinco genotipos de maíz cultivados bajo condiciones de temporal en el centro de Veracruz.

\section{MATERIALES Y MÉTODOS}

Durante el ciclo de temporal 1998B se estableció un experimento en terrenos del Campo Experimental
Cotaxtla (CECOT), perteneciente al Instituto Nacional de Investigaciones Forestales y Agropecuarias (INIFAP), el cual se encuentra ubicado en el municipio de Medellín de Bravo, Veracruz, México, a $18^{\circ} 50^{\prime}$ latitud norte y $96^{\circ} 10^{\prime}$ longitud oeste.

El tipo de clima es cálido subhúmedo Aw" (w)(g) de acuerdo a Köppen modificado por García (1981), con una media anual de temperatura y precipitación de $25^{\circ} \mathrm{C}$ y $1400 \mathrm{~mm}$, respectivamente, y un periodo de lluvias comprendido entre los meses de junio a octubre (Reyes 1994). El tipo de suelo es de origen aluvial, de textura migajón-arcillosa, buen drenaje superficial e interno y pH ligeramente ácido (Tosquy 1997).

El diseño experimental utilizado fue bloques al azar con dos repeticiones con arreglo con parcelas subdivididas. Las parcelas grandes correspondieron a las seis densidades de población (D), las parcelas medianas las conformaron cinco niveles de fertilización (F) y las parcelas chicas fueron cinco genotipos de maíz (G), dos de los cuales son híbridos trilineales promisorios con posibilidades de ser liberados (Cuadro1), resultando un total de 150 tratamientos.

Cuadro 1. Factores en estudio y niveles de exploración en el experimento establecido en el CECOT. México. PV. 1998B.

\begin{tabular}{lccl}
\hline Factor & $\mathbf{D}$ & $\mathbf{F}$ & \multicolumn{1}{c}{$\mathbf{G}$} \\
\hline Unidad & plantas/ha & $\mathrm{N}_{2}, \mathrm{P}_{2} \mathrm{O}_{5}, \mathrm{~K}_{2} \mathrm{O}(\mathrm{kg} / \mathrm{ha})$ & (cualitativos) \\
Dosis & 50000 & $161-46-00(\mathrm{~T})$ & VS-536 (T) \\
& 62500 & $184-69-00$ & $\mathrm{H}-512 \quad(\mathrm{~T})$ \\
& 75000 & $184-69-30$ & $\mathrm{H}-513 \quad(\mathrm{~T})$ \\
& 93750 & $207-92-00$ & HTV-1 * \\
& 100000 & $207-92-30$ & HTV-2 * \\
& 125000 & & \\
\hline
\end{tabular}

$\mathrm{T}:$ Testigo. $\quad *$ : Promisorios.

Se usaron parcelas de dos surcos de cinco metros de longitud, espaciados a $0,80 \mathrm{~m}$ de distancia además para evitar el efecto de orilla, cada parcela media fue separada por bordes de dos surcos. El manejo de cultivo se hizo de acuerdo a las recomendaciones para maíz del Campo Experimental Cotaxtla (Martínez et al., 1990). La cosecha se realizó en forma manual durante el mes de noviembre.

Se realizaron mediciones en planta, mazorca y grano, considerándose como variables de respuesta de índice de cosecha (IC), porcentaje de plantas jorras (\%PJ), el cual para el análisis de varianza fueron transformados los valores según Reyes (1990) a grados Bliss 
$(\sqrt{\mathrm{X}+1})$ y el rendimiento de grano $(\mathrm{RG})$ expresado en t/ha y ajustado al $14 \%$ de humedad.

\section{RESULTADOS Y DISCUSIÓN}

En el Cuadro 2 se presenta el resumen de los análisis de varianza realizados a las diferentes variables evaluadas, donde se visualiza que para el factor D se detectó diferencia altamente significativa para las variables índice de cosecha y porcentaje de plantas jorras (sin mazorcas), lo cual implica que las densidades afectan la magnitud de la expresión fenotípica de los caracteres agronómicos anteriormente expuestos, o sea que responden de manera diferente según la densidad a la que sean sometidos. Para el factor F y la interacción D x F sólo se encontró significancia al $5 \%$ en la variable índice de cosecha, por lo que los valores obtenidos en los parámetros rendimiento de grano y porcentaje de plantas jorras son similares en cada tratamiento de fertilización y los factores densidad y fertilización actúan independientemente. Con respecto a $\mathrm{G}$ se notan diferencias altamente significativas en las tres variables; esto indica el diferente comportamiento que tuvieron los materiales, dado principalmente por la constitución genética de los mismos. La interacción D x G causó significancia estadística al $1 \%$ en el rendimiento de grano e índice de cosecha, las diferencias detectadas para estas características son atribuidas al efecto conjunto causado por estos dos factores. El efecto de las interacciones F x G y D x F x G fue no significativo en todas las variables. Por lo que se concluye que los factores en consideración son independientes entre sí para esos parámetros; es decir los efectos simples de un factor son los mismos para todos los niveles de los otros factores

Cuadro 2. Cuadrados medios y significancia de las variables evaluadas en el ensayo agronómico desarrollado en el CECOT, México. 1998 B.

\begin{tabular}{lrllc}
\hline \multicolumn{1}{c}{ F.V. } & G.L. & \multicolumn{1}{c}{ RG } & \multicolumn{1}{c}{ IC } & \%PJ \\
\hline Repetición & 1 & $13,696 *$ & $0,049946 *$ & $0,236 \mathrm{NS}$ \\
Densidad (D) & 5 & $5,939 \mathrm{NS}$ & $0,073802 * *$ & $51,606 * *$ \\
Error A & 5 & 2,103 & 0,005597 & 1,521 \\
Fertilización (F) & 4 & $0,328 \mathrm{NS}$ & $0,005643 *$ & $0,587 \mathrm{NS}$ \\
D x F & 20 & $0,313 \mathrm{NS}$ & $0,003945 *$ & $0,644 \mathrm{NS}$ \\
Error B & 24 & 0,312 & 0,001462 & 0,936 \\
Genotipo (G) & 4 & $19,997 * *$ & $0,008956 * *$ & $18,477 * *$ \\
D x G & 20 & $1,033 * *$ & $0,005819 * *$ & $1,243 \mathrm{NS}$ \\
F x G & 16 & $0,305 \mathrm{NS}$ & $0,001246 \mathrm{NS}$ & $0,396 \mathrm{NS}$ \\
D x F x G & 80 & $0,277 \mathrm{NS}$ & $0,001347 \mathrm{NS}$ & $0,630 \mathrm{NS}$ \\
Error C & 120 & 0,310 & 0,001320 & \\
Total & 299 & & & \\
\hline
\end{tabular}

C.V. ${ }_{\mathrm{RG}}=9,56 \%$ C.V.V.I. $=10,78 \%$ C.V. $\%$ PJ $=22,84 \%$ dentro de una variación aleatoria medido por el error experimental (Steel y Torrie 1990). Los coeficientes de variación son muy aceptables considerando que el experimento se condujo bajo condiciones de temporal.

La prueba de Tukey al $1 \%$ realizada a las variables índice de cosecha y porcentaje de plantas jorras en cada una de las densidades corroboró las diferencias que existen dándole validez estadística a las medias obtenidas, lo cual se observa en el Cuadro 3, donde el mejor índice de cosecha $(0,39)$ obtenido fue cuando se sembraron $62500 \mathrm{pl} / \mathrm{ha}$ y el más alto porcentaje de plantas jorras $(16,94)$ cuando se utilizaron $125000 \mathrm{pl} / \mathrm{ha}$. Por el contrario, los valores más bajos de índice de cosecha y porcentaje de plantas jorras se encontraron al establecer 125 y 50000 pl/ha, respectivamente. Por otro lado, aunque no se detectó significancia estadística para rendimiento de grano se encontró que el mayor rendimiento $(6,29 \mathrm{t} / \mathrm{ha})$ se obtuvo con $62500 \mathrm{pl} / \mathrm{ha}$, mientras que con 125000 pl/ha se logró la menor cifra. Los análisis de regresión (datos no mostrados) arrojaron diferencia significativa para esta última variable y altamente significativa para índice de cosecha y porcentaje de plantas jorras. Esto significa que efectivamente la densidad tiene un efecto muy marcado en las medias obtenidas en cada una de las variables (coeficientes de determinación de 0,$80 ; 0,87$ y 0,91 , respectivamente), ya que a medida que se incrementa el número de plantas por unidad de área, se incrementa el número de plantas jorras y disminuye el índice de cosecha y el rendimiento de grano.

Cuadro 3. Prueba de Tukey realizada a la variable índice de cosecha en cada uno de los niveles de los factores densidad (D) y fertilización (F). CECOT, México. 1998 B.

\begin{tabular}{rrcrrrrr}
\hline & $\begin{array}{r}\text { Factor } \\
(\mathbf{p l . / h a})\end{array}$ & $\begin{array}{l}\text { D IC } \\
(* *)\end{array}$ & $\begin{array}{c}\text { \% PJ } \\
(* *)\end{array}$ & $\begin{array}{r}\text { Factor F } \\
(\mathbf{k g} / \mathbf{h a})\end{array}$ & $\begin{array}{c}\text { IC } \\
(*)\end{array}$ \\
\hline 1 & 50000 & $0,37 \mathrm{AB}$ & $3,27 \mathrm{~F}$ & 1 & $161-46-00$ & $0,33 \mathrm{~B}$ \\
2 & 62500 & $\bullet 0,39 \mathrm{~A}$ & $5,15 \mathrm{E}$ & 2 & $184-69-00$ & $\bullet 0,35 \mathrm{~A}$ \\
3 & 75000 & $0,34 \mathrm{AB}$ & $7,74 \mathrm{D}$ & 3 & $184-69-30$ & $0,33 \mathrm{~B}$ \\
4 & 93750 & $0,32 \mathrm{AB}$ & $14,97 \mathrm{~B}$ & 4 & $207-92-00$ & $0,34 \mathrm{AB}$ \\
5 & 100000 & $0,31 \mathrm{AB}$ & $12,28 \mathrm{C}$ & 5 & $207-92-30$ & $0,33 \mathrm{~B}$ \\
6 & 125000 & $0,29 \mathrm{~B}$ & $\bullet 16,94 \mathrm{~A}$ & & & \\
\hline
\end{tabular}

- Valores máximos alcanzados en cada variable.

*, ** Las medias de los tratamientos con letras similares son estadísticamente iguales en el nivel de 5 y $1 \%$, respectivamente según Tukey .

En el mismo Cuadro 3 se puede apreciar que con el tratamiento de fertilización 184-69-0 se obtuvo el mayor índice de cosecha con 0,35 , sin embargo, las diferencias fueron mínimas en cada uno de ellos. Referente a la interacción D x F (Cuadro 4) debido a que se observó heterogeneidad de varianza en las medias de los tratamientos de la variable rendimiento de grano se 
Cuadro 4. Prueba de Tukey para la interacción Densidad x Fertilización ( $\mathrm{D} \times \mathrm{F})$ en las variables evaluadas en el ensayo agronómico. CECOT, México. 1998 B.

\begin{tabular}{|c|c|c|c|c|c|}
\hline & Trata & mientos & RG & & IC \\
\hline & D (pl/ha) & F (kg/ha) & $(\mathrm{t} / \mathrm{ha})$ & & \\
\hline & & $\mathrm{N}_{2}, \mathbf{P}_{2} \mathrm{O}_{5}, \mathrm{~K}_{2} \mathrm{O}$ & $* *$ & & * \\
\hline 1 & 50000 & $161-46-00$ & 5,76 А В & 0,362 & BCDEFGH \\
\hline 2 & 50000 & $184-69-00$ & 6,06 А В & 0,398 & $\mathrm{AB}$ \\
\hline 3 & 50000 & $184-69-30$ & 5,92 A B & 0,371 & $\mathrm{BCDE}$ \\
\hline 4 & 50000 & $207-92-00$ & 6,07 A B & 0,362 & BCDEFG \\
\hline 5 & 50000 & $207-92-30$ & 6,19 А В & 0,378 & $\mathrm{ABCD}$ \\
\hline 6 & 62500 & $161-46-00$ & 6,08 A B & 0,366 & BCDEF \\
\hline 7 & 62500 & $184-69-00$ & $6,38 \mathrm{~A}$ & $\bullet 0,446$ & A \\
\hline 8 & 62500 & $184-69-30$ & $\bullet 6,41 \mathrm{~A}$ & 0,373 & ABCDE \\
\hline 9 & 62500 & $207-92-00$ & $6,39 \mathrm{~A}$ & 0,396 & $\mathrm{ABC}$ \\
\hline 10 & 62500 & $207-92-30$ & 6,21 A B & 0,363 & BCDEFG \\
\hline 11 & 75000 & $161-46-00$ & 5,67 A B & 0,325 & BCDEFGH \\
\hline 12 & 75000 & $184-69-00$ & 5,97 А B & 0,333 & BCDEFG \\
\hline 13 & 75000 & $184-69-30$ & 6,04 A B & 0,337 & BCDEFG \\
\hline 14 & 75000 & $207-92-00$ & 5,74 A B & 0,342 & BCDEFG \\
\hline 15 & 75000 & $207-92-30$ & 6,01 A B & 0,354 & BCDEFG \\
\hline 16 & 93750 & $161-46-00$ & 5,86 A B & 0,352 & BCDEFG \\
\hline 17 & 93750 & $184-69-00$ & 6,02 A B & 0,323 & CDEFGH \\
\hline 18 & 93750 & $184-69-30$ & $5,50 \mathrm{AB}$ & 0,302 & EFGH \\
\hline 19 & 93750 & $207-92-00$ & 5,52 A B & 0,319 & DEFGH \\
\hline 20 & 93750 & $207-92-30$ & 5,74 A B & 0,309 & DEFGH \\
\hline 21 & 100000 & $161-46-00$ & 5,87 A B & 0,327 & BCDEFG \\
\hline 22 & 100000 & $184-69-00$ & 5,89 A B & 0,326 & BCDEFGH \\
\hline 23 & 100000 & $184-69-30$ & $5,52 \mathrm{~A} \mathrm{~B}$ & 0,307 & DEFGH \\
\hline 24 & 100000 & $207-92-00$ & 6,05 A B & 0,313 & DEFGH \\
\hline 25 & 100000 & $207-92-30$ & 5,53 A B & 0,290 & $\mathrm{GH}$ \\
\hline 26 & 125000 & $161-46-00$ & 5,14 В & 0,253 & $\mathrm{H}$ \\
\hline 27 & 125000 & $184-69-00$ & 5,29 A B & 0,296 & FGH \\
\hline 28 & 125000 & $184-69-30$ & 5,35 A B & 0,291 & GH \\
\hline 29 & 125000 & $207-92-00$ & 5,18 А В & 0,291 & GH \\
\hline 30 & 125000 & $207-92-30$ & 5,31 A B & 0,306 & DEFGH \\
\hline
\end{tabular}

- Valores máximos alcanzados en cada una de las variables.

*, ** Las medias de los tratamientos con letras similares son estadísticamente iguales en el nivel de 5 y 1\%, respectivamente según Tukey.

realizó la prueba de Tukey al 1\%, donde los mejores tratamientos fueron: 62500 184-69-30, 62500 207-92-0 y 62500 184-69-00 con rendimientos cercanos a las 6,5 t/ha y los más bajos rendimientos $(5,14 ; 5,18$ y 5,29 t/ha) se obtuvieron con los tratamientos: 125000 61-460,125000 207-92-00 y 125, 184-69-0. Un comportamiento muy similar se obtuvo con el índice de cosecha, donde según Tukey al 5\% los tratamientos: $62500184-$ 69-00, 50000 184-69-00 y 62500 207-92-00 arrojaron los valores más altos y con los tratamientos: 125000 $161-46-00,100000$ 207-92-30, 125000 184-69-30 у 125000 207-92-00 se obtuvieron los menores índices de cosecha. En forma general se puede apreciar que después de $62500 \mathrm{pl} / \mathrm{ha}$ a medida que se incrementa la población de plantas disminuye tanto el rendimiento de grano como el índice de cosecha.
Referente a genotipos la prueba de Tukey al $1 \%$ (Cuadro 5) mostró que el mejor material en cuanto a rendimiento de grano fue el híbrido experimental HTV1 seguido del HTV-2, ambos trilineales, cuya cruza simple hembra es la misma que la del híbrido de cruza doble H-512, el cual presentó el mayor índice de cosecha $(0,36)$ y un rendimiento de grano aceptable. Por su parte, el híbrido H-513 obtuvo el menor rendimiento con 5,24 t/ha y el mayor porcentaje de plantas sin producir mazorca $(16,82)$.

Cuadro 5. Prueba de Tukey al $1 \%$ realizadas en las variables estudiadas en cada uno de los genotipos $(\mathrm{G})$. CECOT, México. 1998 B.

\begin{tabular}{llcrr}
\hline & Genotipo & RG (t/ha) & \multicolumn{1}{c}{ IC } & \%PJ \\
\hline 1 & VS-536 & $5,45 \mathrm{CD}$ & $0,33 \mathrm{~B}$ & $9,50 \mathrm{~B}$ \\
2 & H-512 & $5,78 \mathrm{BC}$ & $* 0,36 \mathrm{~A}$ & $8,61 \mathrm{C}$ \\
3 & H-513 & $5,24 \mathrm{D}$ & $0,32 \mathrm{~B}$ & $* 16,82 \mathrm{~A}$ \\
4 & HTV-1 & $* 6,74 \mathrm{~A}$ & $0,34 \mathrm{AB}$ & $7,22 \mathrm{D}$ \\
5 & HTV-2 & $5,90 \mathrm{~B}$ & $0,34 \mathrm{AB}$ & $8,15 \mathrm{C}$ \\
\hline
\end{tabular}

* Valores máximos alcanzados en cada una de las variables.

En el Cuadro 6 se presenta la prueba de Tukey al $1 \%$ para la interacción $\mathrm{D}$ x G, donde se visualiza que el mayor rendimiento de grano $(7,26 \mathrm{t} / \mathrm{ha})$ se logró con el híbrido HTV-1 sembrado a $62500 \mathrm{pl} / \mathrm{ha}$, seguido de este mismo genotipo sembrado a 75 y $50000 \mathrm{pl} / \mathrm{ha}$, los más bajos rendimientos (4,68 y 4,06 t/ha) se obtuvieron utilizando densidades de población de 93750 y 125000 pl/ha con el híbrido H-513. En este sentido, Espinosa y Tadeo (1990) en estudios de fertilización y densidades con las cruzas simples progenitoras del híbrido doble de maíz H-137 concluyeron que la densidad de población que da el mayor rendimiento en ambos cruzas es a $60000 \mathrm{pl} / \mathrm{ha}$. Concerniente al índice de cosecha los mayores valores se dieron cuando los híbridos H-512 y H513 fueron sembrados a 62500 y $50000 \mathrm{pl} / \mathrm{ha}$, respectivamente, y los índices más bajos $(0,235$ y 0,271$)$ se obtuvieron cuando este último híbrido se sembró a 125 y $93750 \mathrm{pl} / \mathrm{ha}$.

La Figura 1 muestra el efecto de la densidad sobre el rendimiento de grano de los genotipos, donde se aprecia que en todos los materiales el mayor rendimiento medio se da al establecer $62500 \mathrm{pl} / \mathrm{ha}$ y que a medida que se aumenta el número de plantas por unidad de área disminuye éste. Por su parte, el híbrido H-513 resultó ser el más inestable, ya que en densidades mayores se vio afectado negativamente más que los demás genotipos. VS-536 aunque no fue sobresaliente resultó ser la variedad más constante. 
Cuadro 6. Prueba de Tukey al $1 \%$ para la interacción Densidad $x$ Genotipo ( $\mathrm{D} \times \mathrm{G}$ ) en el rendimiento de grano e índice de cosecha. CECOT, México. 1998 B.

\begin{tabular}{|c|c|c|c|c|c|c|}
\hline & \multicolumn{2}{|c|}{ Tratamientos } & \multirow{2}{*}{\multicolumn{2}{|c|}{$\begin{array}{c}\text { RG } \\
(\mathbf{t} / \mathbf{h a})\end{array}$}} & \multirow{2}{*}{\multicolumn{2}{|c|}{ IC }} \\
\hline & D (pl/ha) & $\mathbf{G}$ & & & & \\
\hline 1 & 50,000 & VS-536 & 5,55 & DEFGHI & 0,362 & ABCDEF \\
\hline 2 & 50,000 & H-512 & 5,84 & BCDEFGH & 0,372 & $\mathrm{ABCD}$ \\
\hline 3 & 50,000 & H-513 & 6,11 & BCDEFGH & 0,404 & A \\
\hline 4 & 50,000 & HTV-1 & 6,66 & $\mathrm{ABC}$ & 0,364 & ABCDE \\
\hline 5 & 50,000 & HTV-2 & 6,86 & BCDEFGH & 0,369 & $\mathrm{ABCD}$ \\
\hline 6 & 62,500 & VS-536 & 5,72 & CDEFGHI & 0,364 & ABCDE \\
\hline 7 & 62,500 & $\mathrm{H}-512$ & 6,06 & BCDEFGH & $\bullet 0,407$ & A \\
\hline 8 & 62,500 & H-513 & 6,20 & ABCDEFG & 0,395 & $\mathrm{AB}$ \\
\hline 9 & 62,500 & HTV-1 & $\cdot 7,26$ & A & 0,388 & $\mathrm{ABC}$ \\
\hline 10 & 62,500 & HTV-2 & 6,23 & ABCDEFG & 0,390 & $\mathrm{ABC}$ \\
\hline 11 & 75,000 & VS-536 & 5,34 & GHI & 0,300 & DEFGHI \\
\hline 12 & 75,000 & H-512 & 5,76 & BCDEFGHI & 0,341 & ABCDEFGH \\
\hline 13 & 75,000 & H-513 & 5,35 & GHI & 0,346 & ABCDEFG \\
\hline 14 & 75,000 & HTV-1 & 6,85 & $\mathrm{AB}$ & 0,360 & ABCDEF \\
\hline 15 & 75,000 & HTV-2 & 6,13 & BCDEFGH & 0,344 & ABCDEFG \\
\hline 16 & 93,750 & VS-536 & 5,48 & EFGHI & 0,319 & CDEFGH \\
\hline 17 & 93,750 & $\mathrm{H}-512$ & 5,84 & BCDEFGH & 0,364 & ABCDE \\
\hline 18 & 93,750 & H-513 & 4,68 & $\mathrm{IJ}$ & 0,271 & HI \\
\hline 19 & 93,750 & HTV-1 & 6,56 & $\mathrm{ABCDE}$ & 0,310 & DEFGH \\
\hline 20 & 93,750 & HTV-2 & 6,08 & BCDEFGH & 0,341 & ABCDEFGH \\
\hline 21 & 100,000 & VS-536 & 5,42 & FGHI & 0,330 & BCDEFGH \\
\hline 22 & 100,000 & $\mathrm{H}-512$ & 5,80 & BCDEFGH & 0,342 & ABCDEFGH \\
\hline 23 & 100,000 & H-513 & 5,03 & HIJ & 0,291 & FGHI \\
\hline 24 & 100,000 & HTV-1 & 6,63 & $\mathrm{ABCD}$ & 0,305 & DEFGHI \\
\hline 25 & 100,000 & HTV-2 & 5,99 & BCDEFGH & 0,295 & EFGHI \\
\hline 26 & 125,000 & VS-536 & 5,22 & GHI & 0,313 & DEFGH \\
\hline 27 & 125,000 & $\mathrm{H}-512$ & 5,35 & GHI & 0,314 & DEFGH \\
\hline 28 & 125,000 & H-513 & 4,06 & $\mathrm{~J}$ & 0,235 & I \\
\hline 29 & 125,000 & HTV-1 & 6,50 & $\mathrm{ABCDEF}$ & 0,292 & EFGHI \\
\hline 30 & 125,000 & HTV-2 & 5,14 & GHIJ & 0,283 & GHI \\
\hline
\end{tabular}

* Valores máximos alcanzados en cada una de las variables Las medias de los tratamientos con letras similares son estadísticamente iguales según Tukey.

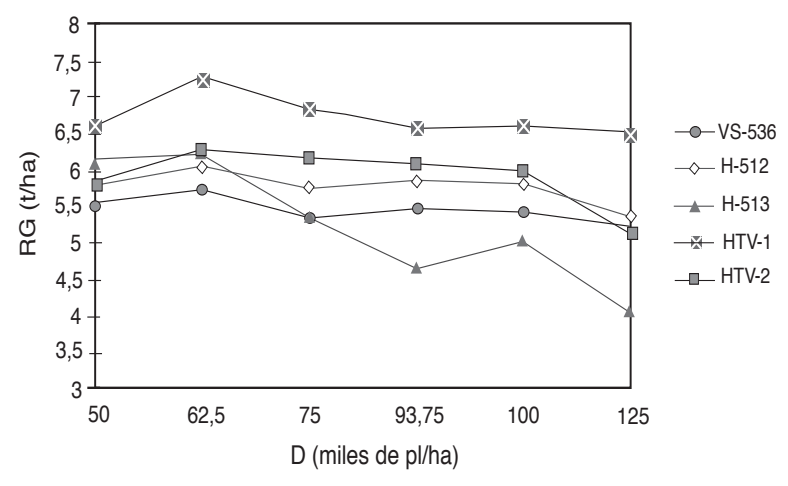

Figura 1. Efecto de la densidad y genotipo sobre el rendimiento en grano. CECOT, México. 1998 B.

\section{CONCLUSIONES}

Los resultados encontrados permiten derivar las siguientes conclusiones:

La densidad que aportó el mayor rendimiento de grano e índice de cosecha fue $62500 \mathrm{pl} / \mathrm{ha}$, la cual presentó un bajo porcentaje de plantas "jorras".

No hubo respuesta para rendimiento de grano y porciento de plantas jorras a los tratamientos de fertilización.

El mejor genotipo para rendimiento de grano fue el híbrido experimental HTV-1 con 6,74 t/ha, que superó en $1,5 \mathrm{t} / \mathrm{ha}$ al testigo comercial H-513.

\section{LITERATURA CITADA}

CASTAÑÓN, N.; SIERRA, M.; TOSQUY, O; RODRÍGUEZ, F. 1996. Dosis óptima económica de N-P-K en híbridos de temporal. In: Memoria del XVI Congreso de Fitogenética. Montecillo, Texcoco. Edo. de México. p. 342.

ESPINOSA, C.A.; TADEO, M.R. 1990. Tecnología de producción de semillas del híbrido de cruza doble de maíz H-137 de Valles Altos. In: Resúmenes del XIII Congreso Nacional de Fitogenética. Escuela Superior de Agricultura "Hermanos Escobar”, Cd. Juárez, Chihuahua, México. p. 370.

GARCÍA, E. 1981. Modificaciones al sistema de clasificación Climática de Köppen. Tercera ed. 252 p.

INPOFOS (s.f.). Potasa: su necesidad y uso en agricultura moderna. Quito, Ecuador. p. 9-11.

MARÍN, G.; RIESTRA, O.; EXEBIO, G.A.; MARTÍNEZ, G.A. 1989. Respuesta del maíz CP-561 a presión poblacional, nitrógeno y balance hídrico bajo temporal en la Región Central Costera de Veracruz. Agrociencia (Méx.) 78: 79-98.

MARTÍNEZ, J.; SIERRA, M.; PRECIADO, R.E.; AGUILAR, J.L.; RODRÍGUEZ, F.; TINOCO, A.C.; ARROYO, M.C. 1990. Manual de producción de maíz en el estado de Veracruz. Folleto para productores Núm. 5 SARH. INIFAP. CIFAP. Veracruz, México. 23 p.

REYES, C.P. 1990. Diseño de experimentos aplicados. Trillas. México, D.F. p. 229-300.

REYES, V.A.S. 1994. Evaluación agronómica de híbridos experimentales de maíz (Zea mays L.) de cruza simple bajo condiciones de riego. Tesis Profesional. Instituto Tecnológico Agropecuario No.18. Ursulo Galván, Veracruz, México. p. 24.

STEEL, R.; TORRIE, J. 1990. Bioestadística: principios y procedimientos. Mc. Graw-Hill. México, D.F. p. 332-345.

TOSQUY, O. 1997. Tecnología para la producción de semilla de líneas que forman híbridos de maíz tropicales. Tesis de Maestría en Ciencias en Suelos. Programa de graduados. Universidad Autónoma Agraria Antonio Narro, Saltillo, Coah., México. p. 2, 20. 